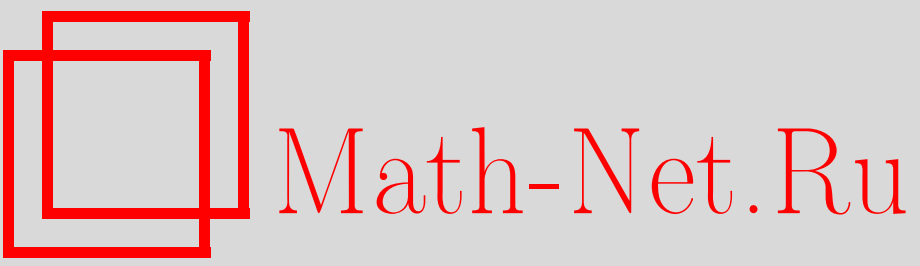

П. С. Бачурин, О связи между временными средними и минимальными аттракторами, УМH, 1999, том 54, выпуск 6, 151-152

DOI: https://doi.org/10.4213/rm232

Использование Общероссийского математического портала Math-Net.Ru подразумевает, что вы прочитали и согласны с пользовательским соглашением

http://www . mathnet.ru/rus/agreement

Параметры загрузки:

IP: 54.172 .240 .79

26 апреля 2023 г., 06:41:34 


\title{
О СВЯЗИ МЕЖДУ ВРЕМЕННЫМИ СРЕДНИМИ И МИНИМАЛЬНЫМИ АТТРАКТОРАМИ
}

\author{
П.С. БАчУРИн
}

Известно [1], что для гиперболического аттрактора $\Omega$, на котором диффеоморфизм $f$ класса $C^{2}$ действует транзитивно, существует и единственна инвариантная мера $\mu$ такая, что

$$
\forall \varphi \in C(\Omega) \quad \lim _{n \rightarrow \infty} \frac{1}{n} \sum_{i=0}^{n-1} \varphi \circ f^{i}=\int \varphi d \mu .
$$

Пусть $B$ - компакт, вложенный в $\mathbb{R}^{n}$ и имеющий положительную меру Лебега. Рассмотрим диффеоморфизм класса $C^{r}, r \geqslant 1$, компакта $B$ строго внутрь себя: $f: B \rightarrow B, f(B) \subset B$. Пусть $m$ - ограничение меры Лебега на $B$. Существуют различные определения притягивающего множества отображения $f$ : максимальный аттрактор, множество неблуждающих точек, предельное множество, аттрактор Милнора и т.д. Один из способов определить аттрактор состоит в использовании временных средних непрерывных на $B$ функций.

Основной целью работы является доказательство того, что временные средние непрерывных функций зависят только от ограничения этих функций на минимальный аттрактор. Временные средние для динамических систем без заданной априори инвариантной меры определяются ниже. Напомним определение минимального аттрактора [2]-[4].

ОпредЕЛЕниЕ 1. Открытое множество $U$ называется существенным, если существуют последовательность $k_{n} \rightarrow \infty$ и $\varepsilon>0, \delta>0$ такие, что

$$
m\left\{x \in B: \frac{1}{k_{n}} \sum_{i=0}^{k_{n}-1} \chi_{u} \circ f^{i}(x)>\varepsilon\right\}>\delta \quad \forall n \in \mathbb{N} .
$$

ОпРЕДЕлЕниЕ 2. Минималњным аттрактором называется множество:

$$
A_{\min }=B \backslash\{\bigcup W: W \text { открыто и не является сушественным }\} .
$$

Другое определение минимального аттрактора можно получить, если рассматривать предельные точки последовательности усредненных итераций меры Лебега: $\mu_{t}=\frac{1}{t} \sum_{i=0}^{t-1} f^{i} m$. Пространство вероятностных мер на $B$ компактно в слабой* топологии. Следовательно, множество предельных точек последовательности $\mu_{t}$ непусто.

ОпрЕдЕЛЕниЕ 3. Предельные точки последовательности $\mu_{t}$ назьваются "хорошими" мерами.

"Хорошие" меры инвариантны. Будем обозначать их $\mu_{\infty}$.

ОпредЕлЕниЕ 4. $A_{\min }^{g}=\overline{\bigcup \operatorname{supp} \mu_{\infty}}$, объединение ведется по носителям всех "хороших" мер.

В [4] доказана следующая теорема.

Tеорема 1. $A_{\min }$ совпадает с множеством $A_{\min }^{g}$.

Для непрерывной на компакте $B$ неотрицательной функции $\varphi$ рассмотрим последовательность временных средних $\varphi_{n}=\frac{1}{n} \sum_{i=0}^{n-1} \varphi \circ f^{i}$. Из последовательности мер $\varphi_{n} m$ на $B$ можно выбрать сходящуюся в слабой* топологии подпоследовательность. Обозначим предельную меру через $\varphi_{\infty} \mathrm{m}$. Рассмотрим ограниченный линейный функционал $\varphi_{\infty}$ на пространстве непрерьвных на $B$ функций, который действует по формуле: $\left(\varphi_{\infty}, \psi\right)=\int \psi d\left(\varphi_{\infty} m\right)$. Функционал $\varphi_{\infty}$ называется слабым временным средним функции $\varphi$.

Основное утверждение работы состоит в том, что слабое временное среднее непрерывной функиии зависит только от ограничения исходной функчии на минимальный аттрактор, и в любой окрестности любой точки минимального аттрактора непрерывнуло функцию можно изменить так, что изменится одно из ее временных средних.

ЗАмечАние 1. Для каждой непрерывной функции $\varphi$ на $B$ существует "хорошая" мера (в смысле определения 3$) \mu_{\infty}$ такая, что $\left(\varphi_{\infty}, 1\right)=\int \varphi d \mu_{\infty}$.

Работа выполнена при поддержке Российского фонда фундаментальных исследований (грант № 98-01-00455). 
Пусть $\psi \geqslant 0, \psi \in L^{\infty}(m)$. Рассмотрим последовательность усредненных итераций меры $\psi m$. Ее предельные точки будем обозначать через $\mu_{\psi}$. Если в определении 2 заменить меру $m$ на меру $\psi m$, то получится определение инвариантного множества, которое будем обозначать $A_{\min }(\psi m)$. Та же замена в определении 4 приводит к инвариантному множеству $A_{\min }^{\prime}(\psi m)$, для которого справедлив аналог теоремы 1:

Teopema $1^{\prime} . A_{\min }(\psi m)=A_{\min }^{\prime}(\psi m)$.

Лемма 1. $A_{\min }(\psi m) \subseteq A_{\min }$.

ДокАЗАТЕльство. Каждое существенное множество для меры $\psi m$ является существенным для $m$, так как если $|\psi|<C$ (m-почти всюду) и $\int_{A} \psi d m \geqslant \delta$, то $m(A) \geqslant \delta / C$. Поэтому $\{\bigcup W: W$ открыто и не является существенным для $m\} \subseteq\{\bigcup W: W$ открыто и не является существенным для $\psi m\}$. Лемма следует из определения 2.

Замечание 2 . Если $\psi \geqslant q>0$ для некоторого $q$, то $A_{\min }(\psi m)=A_{\min }$.

Теорема 2. а) Носитель слабого временного среднего любой непрерывной на В функиии лежит в минимальном аттракторе.

б) Для любой непрерывной на $B$ функчии $\varphi$, любого открытого множества $U$, имеющего непустое пересечение с минимальным аттрактором, и для любого $\varepsilon>0$ существует непрерывная на $B$ функиия $\varphi^{\prime}$ такая, что $\left\|\varphi-\varphi^{\prime}\right\|_{C(B)}<\varepsilon, \varphi=\varphi^{\prime}$ вне $U$ и существуют слабъе временные средние $\varphi_{\infty}$ и $\varphi_{\infty}^{\prime}$ функиий $\varphi$ и $\varphi^{\prime}$ такие, что $\left(\varphi_{\infty}, 1\right) \neq\left(\varphi_{\infty}^{\prime}, 1\right)$.

ДокАЗАТЕльСтво. а) Без ограничения общности будем считать, что $\psi \geqslant 0$. Тогда

$$
\left(\psi, \varphi_{\infty}\right)=\lim _{n \rightarrow \infty} \int \frac{1}{k_{n}} \sum_{i=0}^{k_{n}-1} \varphi \circ f^{i} d(\psi m), \quad \int \frac{1}{k_{n}} \sum_{i=0}^{k_{n}-1} \varphi \circ f^{i} d(\psi m)=\int \varphi d \widetilde{\mu}_{k_{n}},
$$

где $\tilde{\mu}_{k_{n}}=\frac{1}{k_{n}} \sum_{i=0}^{k_{n}-1} f^{i}(\psi m)$. Выберем из последовательности $\tilde{\mu}_{k_{n}}$ сходящуюся подпоследовательность; пусть $\mu_{\psi}$ - ее предел. Получим $\left(\psi, \varphi_{\infty}\right)=\int \varphi d \mu_{\psi}$. По лемме $2 \operatorname{supp} \mu_{\psi} \subset A_{\min }$, следовательно, п. а) доказан.

б) Сформулируем сначала следующую лемму.

ЛЕмма 2. Для любого существенного множества U существует “хорошая" мера $\mu_{\infty}$ такая, что $\mu_{\infty}(U)>0$.

Доказательство леммы 2 содержится в доказательстве теоремы 1.

Пусть $U \cap A_{\min } \neq \varnothing, \varphi$ - заданная непрерывная на $B$ функция. Выберем $\varphi^{\prime}$ так, что $\varphi^{\prime}(x)>$ $\varphi(x)$ для всех $x \in U$ и $\varphi^{\prime}(x)=\varphi(x)$ для всех $x \in B \backslash U$. Окрестность $U$ является существенной, так как иначе $U \cap A_{\min }=\varnothing$. Из леммы 2 следует, что можно выбрать "хорошую" меру $\mu_{\infty}^{0}$ такую, что $\mu_{\infty}^{0}(U)>0$. Мере $\mu_{\infty}^{0}$ соответствует последовательность $k_{n}: \mu_{k_{n}}$ сходятся к $\mu_{\infty}^{0}$ в слабой* топологии. Из последовательности $\varphi_{k_{n}}=\frac{1}{k_{n}} \sum_{i=0}^{k_{n}-1} \varphi \circ f^{i}$ выберем сходящуюся в слабой* топологии подпоследовательность. Обозначим через $\varphi_{\infty} m$ предельную меру. Тогда из замечания $1\left(\varphi_{\infty}, 1\right)=\int \varphi d \mu_{\infty}^{0}$. Аналогично, выбирая из последовательности $\varphi_{k_{n}}^{\prime}=\frac{1}{k_{n}} \sum_{i=0}^{k_{n}-1} \varphi^{\prime} \circ f^{i}$ сходящуюся в слабой* топологии подпоследовательность, получим меру $\varphi_{\infty}^{\prime} m .\left(\varphi_{\infty}^{\prime}, 1\right)=\int \varphi^{\prime} d \mu_{\infty}^{0}$ и, следовательно, $\left(\varphi_{\infty}, 1\right)<\left(\varphi_{\infty}^{\prime}, 1\right)$. Теорема 2 полностью доказана.

Автор благодарит Ю. С. Ильяшенко за постановку задачи и постоянную поддержку.

\section{СПИСОК ЛИТЕРАТУРЫ}

[1] Bowen R. Equilibrium States and the Ergodic Theory of Anosov Diffeomorphisms. Berlin: Springer-Verlag, 1975 (Lecture Notes in Math. V. 470.) [2] Динамические системы. T. 5. [3] Ilyashenko Yu.S. // Chaos. 1991. V. 1. № 2. P. 168-173. [4] Gorodetski A.S., Ilyashenko Yu.S. // Internat. J. Bifur. Chaos Appl. Sci. Engrg. 1996. V. 6. №6. P. 1177-1183. 